\title{
Immediate Implantation in Maxillary Molar Sites: A Literature Review
}

\author{
Zeinab Rezaei Esfahrood ${ }^{1}$; Maryam Nourelahi ${ }^{2,}$ \\ ${ }^{1}$ Department of Periodontics, School of Dentistry, Shahid Beheshti University of Medical Sciences, Tehran, Iran \\ ${ }^{2}$ Department of Periodontics, School of Dentistry, Semnan University of Medical Sciences, Semnan, Iran \\ ${ }^{*}$ Corresponding author: Maryam Nourelahi, Department of Periodontics, School of Dentistry, Semnan University of Medical Sciences, Semnan, Iran. Tel: +98-9153011606, E-mail: \\ m.noorelahi@gmail.com
}

Received: June 8, 2015; Revised: July 17, 2015; Accepted: July 26, 2015

\begin{abstract}
Context: For many years, the standard protocol for implant placement was the delayed method which is to wait until the bone is matured after tooth extraction.

Evidence Acquisition: The increasing demand for shorter rehabilitation times have led to the introduction of immediate implant placement. Although this method offers many advantages like minimal number of surgical procedures, less morbidity and shorter rehabilitation time, it is associated with some complications such as complicated extractions, localized bone defects surrounding the implant, and improper implant position because of the socket anatomy.

Results: Immediate implants were most frequently used for incisor and premolar sites and there is very little data available on immediate implantation in the molar area. In this research, we reviewed all the existing papers on immediate implantation of maxillary molars with special focus on success and survival rate. The success and survival rate of immediate implantation into fresh extraction socket of maxillary molars was more than $90 \%$, which was similar to conventional type delayed implants.

Conclusions: It was observed that the success and survival rate of maxillary molar immediate implant was relatively high and similar to those of conventional delayed implants.
\end{abstract}

Keywords: Survival Rate; Success Rate; Immediate Implantation; Maxillary Molar

\section{Context}

For many years after the introduction of osseointegration, the standard protocol for implant placement was to wait until the bone is matured after tooth extraction. The reason for this protocol was to reduce the rate of infection and provide adequate bands of keratinized tissue at the time of implant placement. The increasing demand for shorter rehabilitation times have led to the introduction of immediate implant placement. Implant placement in fresh extraction sockets was first discussed in 1970s and has been reviewed extensively since then. Immediate implant placement is associated with several complications including complicated extractions, localized bone defects surrounding the implant, improper implant position because of the socket anatomy, insufficient distance from the adjacent teeth, sockets or implants, and complicated flap closure and barrier membrane placement. On the other hand, immediate implant approach offers some important advantages. It does not require several surgical procedures and results in less morbidity and shorter rehabilitation time (1).

Immediate implants have been most frequently used for incisor and premolar sites and there is very little data available on immediate implantation in the molar area (2).

\subsection{Objective}

The purpose of this study was to evaluate the survival and success rate of implants immediately placed in the maxillary posterior area by the review of data from human research.

\section{Evidence Acquisition}

An electronic search (MEDLINE and Cochrane Oral Health Group Specialized Trials Register) as well as a manual search were performed to detect studies concerning maxillary molar replacements by means of dental implants immediately placed into fresh extraction sockets from 1990 to 2015.

The inclusion criteria of the studies were as follows:

1. Studies on immediate implantation in maxillary molar area, which is defined as implantation at the same time as tooth extraction;

2. Prospective or retrospective human researches only;

3. The success or survival rates of the implantation reported within a follow-up period of at least one year.

Only eight studies fulfilled the criteria. The success or survival rates of immediate implants reported in the studies were evaluated and compared with those of delayed implants.

Copyright (C) 2015, Semnan University of Medical Sciences. This is an open-access article distributed under the terms of the Creative Commons Attribution-NonCommercial 4.0 International License (http://creativecommons.org/licenses/by-nc/4.0/) which permits copy and redistribute the material just in noncommercial usages, provided the original work is properly cited. 


\section{Results}

A brief summary of the studies is presented below:

In a study by Artzi et al. (3), 12 wide-diameter implants were placed in maxillary fresh extraction sockets of first or second molar. An osteotome technique sinus elevation was carried out in 10 sites. Self-tap implants were then inserted into the osteotomy site. Soft tissue healing was uneventful. Osseointegration was observed in radiographs and prosthetic rehabilitation was completed after six months. All the implants showed clinical stability with a survival rate of $100 \%$. Radiographically, no crestal bone resorption was observed around the implant neck.

Acocella et al. (2) in 2010 used a modified technique for implant placement into fresh extraction socket in the first maxillary molar sites. Prosthetic reconstruction was initiated three months later. The implants were followed for 36 months; the results demonstrated cumulative survival rate of $97.96 \%$.

Another study evaluated localized management of the sinus floor technique (LMSF) for implant placement in fresh extraction molar sockets (4). A survival rate of $100 \%$ was reported after a 4-17-year follow up period (mean: 9.78). The implants were stable over time, reporting a mean value of $8.01 \mathrm{~mm}$ at the 13-year follow-up.

Dimensional ridge alterations following immediate implant placement in molar extraction sites was evaluated in a study by Matarasso et al. (5) in 2009. The survival rate of implants after six months was $100 \%$. It was observed in surgical re-entry that the gap between the internal socket wall and the implant surface was filled with bone.

In a study by Fugazzotto (6), the success and failure rates of 137 immediate implants in molar fresh extraction socket with simultaneous sinus floor elevation were evaluated. The follow-up period was up to three years. The interradicular bone was prepared for implant placement by means of a modified trephine and osteotome technique. The cumulative success rate was $97.8 \%$.

Thirty four maxillary first and second molars were extracted in a study by Talebi Ardakani et al. (7) from 2002 to 2008. Implants were inserted in interradicular bone to provide primary stability for the implants. The teeth with any pathologic lesion at the tooth apex or furcation area and the presence of active purulence or fistula were excluded from the study. The maxillary seven-year cumulative survival rates were $92.7 \%$.

Cafiero et al. (8) in 2008 used tapered implants to immediately replace 86 maxillary molars. Molars with acute periapical pathology were excluded. The follow-up period was 12 months. Primary stability was achieved for all the implants. No postsurgical complications were observed. All the implants healed uneventfully, yielding a survival rate of $100 \%$ and healthy soft tissue conditions after 12 months.

Schwartz-Arad et al. (9) in 2000 evaluated the clinical effects of dental implants placed immediately into fresh extraction sites of molar teeth. The mean follow-up pe- riod was 15 months. Cumulative survival rate was $82.3 \%$ during the five-year follow-up period. The survival rate among males was $84 \%$ compared to $93.5 \%$ among females. It was relatively lower in smokers.

The success and survival rate of immediate implantation into fresh extraction socket of maxillary molars was more than $90 \%$, which was similar to conventional type delayed implants.

\section{Conclusions}

Several classifications have been proposed for the timing of implant placement into fresh extraction sockets. The classification of Wilson and Weber includes immediate (same time as extraction), recent (30 to 60 days after the extraction), delayed (after hard tissue maturation), and mature (bone is mature at presentation), to describe the timing of implant placement in relation to soft and hard tissue healings following tooth extraction (10). A new classification for implant placement was assumed in 2004 based on hard and soft tissue changes which included types 1 to 4 (11). Most of the studies described immediate implant placement as placing an implant immediately following tooth extraction. The exceptions were Schropp et al. (12), who defined immediate implantation as implants placed between 3 - 15 days (mean: 10 days) following tooth extraction and Gomez-Roman et al. (13) 1997, who defined it as occurring between 0 - 7 days afterward.

During the past decade, most evidences described immediate dental implant placement as a successful and predictable treatment modality when the sites were carefully selected $(2,14-16)$. Several advantages have been reported to include reduction in the time of edentulism for patients. It has also been suggested that preservation of the bone at the extraction socket may be achieved. We are also allowed to use an implant with maximum length beyond the apex of the extracted tooth to achieve stability, which increases the bone-implant contact surface. When implants are placed in fresh extraction socket, restoration contours are more easily formed to conform to the previously occupying tooth, which is extremely important for restorations in the esthetic zone. It also permits the screw access opening to be in the central fossa, which helps to decrease the failure rate associated with porcelain fracture (17). There is an extra advantage in the maxillary posterior region, which is implant insertion before the pneumatization phenomenon occurs. Molar extraction induces greater pneumatization than premolar extraction, probably because of the larger defect left in the alveolar cavity, which can cause great limitation for selecting an ideal implant length (4). Interestingly, early placement (immediate and earlier delayed) showed consistently better reduction of dehiscence defects than did late implantation in healed alveolar ridges (18).

On the other hand, immediate implant insertion in maxillary molar extraction sockets raises a series of chal- 
lenges for clinicians. The most important problem is related to the difficulty in achieving primary stability in a fresh extraction socket in the posterior maxilla. Initial implant mobility is, in fact, an important factor associated with implant early failure (19).

The interseptal bone must be preserved as much as possible at the time of tooth removal, which requires an atraumatic procedure for tooth extraction, which is sometimes hard to achieve. There is also the often problematic position of the maxillary sinus around the roots of the tooth to be extracted. Other difficulties are the compromised nature of the residual inter-radicular bone in case of periodontal diseases and the difficulty in placing the implant in the prosthetically driven position as a result of the position of the residual interradicular bone. The placement of an implant in one of the three existing root sockets after maxillary molar removal may compromise the implant emergence profile. It also may cause significant off angle loading and the creation of a cantilever effect buccally, mesially, or distally, depending on which extraction socket is chosen to accept the implant (2).

The long term success of endosseous implants placed in both the maxillary and mandibular posterior region is inferior to other areas, which is because of a less than ideal bone quality, especially in posterior maxilla, and greater occlusal loads and wider occlusal table, resulting in mesiodistal and buccolingual cantilever and off axial forces (20). Posterior jaw quadrants provide the area of greatest occlusal need and force, determined in one study to be 82.0 Newton in the molar area versus $61.4 \mathrm{~N}$ in the premolar area (21).

Despite the complications mentioned above, in this review, we found out that sufficient evidence exists to support long-term success and survival rate for implants placed in the fresh socket of maxillary molars. In a retrospective study by Penarrocha et al. (22), the same result was achieved. They compared immediate and delayed implants in the maxillary molar region and found similar success rates. In another retrospective case series by Annibali et al. (23) immediate, early, and late implant placement in first-molar sites were compared and marginal bone loss and soft tissue parameters did not differ significantly among them.

However, it usually requires different modifications in surgical approaches and advancing. For instance, in a study by Artzi et al. (3), the intra-radicular residual bone was entirely drilled during the implant site preparation; thus, bone-to-implant contact could only be obtained by basal bone anchorage. Therefore, a wide-body implant configuration enhanced the chance of initial stability. While some researchers have achieved comparable results $(24,25)$, others have shown a reduced success rate $(26,27)$.

Different techniques of sinus management have also been introduced. For example, the results reported by Acocella et al. (2) and Bruschi et al. (4) with the use of a modification of the Summers' technique or localized management of sinus floor (LMSF) demonstrate a high degree of predictability in the placement of implants in the ideal prosthetic positions at the time of the removal of maxillary molars. In this technique, tapered-end osteotomes with increasing diameters were used in an area of the inter-radicular bone pointed with a round bur. The bone was compressed and imploded beneath the osteotome tip which corresponded to the drilling sequence for the implant to be placed. The osteotomes were also malleted to lift the floor of the sinus. The engagement of the sinus floor with the apex of the implant as well as inserting the implants into the compressed bone helped in gain of primary stability.

Since there is a clinical correlation between implant failure and periodontitis as a reason for tooth extraction (25), in the studies reviewed in this research, none of the implants were placed in fresh extraction sockets of the teeth with periodontal or periapical infection $(2,4,5,7,8)$. Data from human studies in this review suggested that immediate implant placement in maxillary molar extraction sockets appear to be a predictable procedure if proper case selection is conducted.

\section{Acknowledgements}

We thank Mr. Mahdi Tohidian who helped us through the research process.

\section{Funding/Support}

This study was supported by the Deputy of Research, Semnan University of Medical Sciences.

\section{References}

1. Wilson TJ, Roccuzzo M, Ucer C, Beagle JR. Immediate placement of tapered effect (TE) implants: 5-year results of a prospective, multicenter study. Int J Oral Maxillofac Implants. 2013;28(1):261-9.

2. Acocella A, Bertolai R, Sacco R. Modified insertion technique for immediate implant placement into fresh extraction socket in the first maxillary molar sites: a 3-year prospective study. Implant Dent. 2010;19(3):220-8.

3. Artzi Z, Parson A, Nemcovsky CE. Wide-diameter implant placement and internal sinus membrane elevation in the immediate postextraction phase: clinical and radiographic observations in 12 consecutive molar sites. Int J Oral Maxillofac Implants. 2003;18(2):242-9.

4. Bruschi GB, Crespi R, Cappare P, Bravi F, Bruschi E, Gherlone E. Localized management of sinus floor technique for implant placement in fresh molar sockets. Clin Implant Dent Relat Res. 2013;15(2):243-50.

5. Matarasso S, Salvi GE, Iorio Siciliano V, Cafiero C, Blasi A, Lang NP. Dimensional ridge alterations following immediate implant placement in molar extraction sites: a six-month prospective cohort study with surgical re-entry. Clin Oral Implants Res. 2009;20(10):1092-8.

6. Fugazzotto PA. Immediate implant placement following a modified trephine/osteotome approach: success rates of 116 implants to 4 years in function. Int J Oral Maxillofac Implants. 2002;17(1):113-20.

7. Talebi Ardakani MR, Alizadeh Tabari Z, Golami GA, Farahmand AH, Kadkhodazadeh M. Immediate implantation in the maxillary and mandibular molar fresh sockets: technique and results. JPeriodontol Implant Dentistry. 2011;2(2):51-5.

8. Cafiero C, Annibali S, Gherlone E, Grassi FR, Gualini F, Magliano A, 
et al. Immediate transmucosal implant placement in molar extraction sites: a 12-month prospective multicenter cohort study. Clin Oral Implants Res. 2008;19(5):476-82.

9. Schwartz-Arad D, Gulayev N, Chaushu G. Immediate versus nonimmediate implantation for full-arch fixed reconstruction following extraction of all residual teeth: a retrospective comparative study.J Periodontol. 2000;71(6):923-8.

10. Wilson J, Thomas G, Weber HP. Classification of and therapy for areas of deficient bony housing prior to dental implant placement. Int J Periodontics Restor Dent. 1993;13:451-9.

11. Hammerle $\mathrm{CH}$, Chen ST, Wilson TJ. Consensus statements and recommended clinical procedures regarding the placement of implants in extraction sockets. Int J Oral Maxillofac Implants. 2004;19 Suppl:26-8.

12. Schropp L, Kostopoulos L, Wenzel A. Bone healing following immediate versus delayed placement of titanium implants into extraction sockets: a prospective clinical study. Int J Oral Maxillofac Implants. 2003;18(2):189-99.

13. Gomez-Roman G, Schulte W, d'Hoedt B, Axman-Krcmar D. The Frialit-2 implant system: five-year clinical experience in singletooth and immediately postextraction applications. Int J Oral Maxillofac Implants. 1997;12(3):299-309.

14. Bartee BK. Extraction site reconstruction for alveolar ridge preservation. Part 1: rationale and materials selection. J Oral Implantol. 2001;27(4):187-93.

15. Kan JY, Shiotsu G, Rungcharassaeng K, Lozada JL. Maintaining and attenuating periodontal tissues for aesthetic implant placement. J Oral Implantol. 2000;26(1):35-41.

16. Huys LW. Replacement therapy and the immediate post-extraction dental implant. Implant Dent. 2001;10(2):93-102.

17. Lazzara RJ. Transplantation of a preosseointegrated implant from the mental area to a maxillary sinus graft. Int J Periodontics Restorative Dent.1995;15(6):538-47.

18. Chen ST, Wilson TJ, Hammerle CH. Immediate or early placement of implants following tooth extraction: review of biologic basis, clinical procedures, and outcomes. Int J Oral Maxillofac Implants. 2004;19 Suppl:12-25.

19. Polizzi G, Grunder U, Goene R, Hatano N, Henry P, Jackson WJ, et al. Immediate and delayed implant placement into extraction sockets: a 5-year report. Clin Implant Dent Relat Res. 2000;2(2):93-9.

20. Schwartz-Arad D, Grossman Y, Chaushu G. The clinical effectiveness of implants placed immediately into fresh extraction sites of molar teeth.J Periodontol. 2000;71(5):839-44.

21. Bahat O. Treatment planning and placement of implants in the posterior maxillae: report of 732 consecutive Nobelpharma implants. Int J Oral Maxillofac Implants. 1993;8(2):151-61.

22. Penarrocha-Oltra D, Demarchi CL, Maestre-Ferrin L, Penarrocha-Diago M, Penarrocha-Diago M. Comparison of immediate and delayed implants in the maxillary molar region: a retrospective study of 123 implants. Int J Oral Maxillofac Implants. 2012;27(3):604-10.

23. Annibali S, Bignozzi I, Iacovazzi L, La Monaca G, Cristalli MP. Immediate, early, and late implant placement in first-molar sites: a retrospective case series. Int J Oral Maxillofac Implants. 2011;26(5):1108-22.

24. Renouard F, Arnoux JP, Sarment DP. Five-mm-diameter implants without a smooth surface collar: report on 98 consecutive placements. Int J Oral Maxillofac Implants. 1999;14(1):101-7.

25. Polizzi G, Rangert B, Lekholm U, Gualini F, Lindstrom H. Branemark System Wide Platform implants for single molar replacement: clinical evaluation of prospective and retrospective materials. Clin Implant Dent Relat Res. 2000;2(2):61-9.

26. Ivanoff CJ, Grondahl K, Sennerby L, Bergstrom C, Lekholm U. Influence of variations in implant diameters: a 3- to 5-year retrospective clinical report. Int J Oral Maxillofac Implants. 1999;14(2):173-80.

27. Eckert SE, Meraw SJ, Weaver AL, Lohse CM. Early experience with Wide-Platform Mk II implants. Part I: Implant survival. Part II: Evaluation of risk factors involving implant survival. Int J Oral Maxillofac Implants. 2001;16(2):208-16. 